\title{
Prevalence of Septum Pellucidum Variations: A Retrospective Study
}

\author{
Hale Oktem ${ }^{1}$, Alper Dilli², Ayla Kurkcuoglu1, Can Pelin' ${ }^{1}$ \\ ${ }^{1}$ Department of Anatomy, Faculty of Medicine, Baskent University, Ankara, Turkey \\ ${ }^{2}$ Department of Radiology, Ministry of Health, Dişkapi Yildirim Beyazit Training and Research Hospital, Ankara, Turkey \\ Email: ^haleoktem@gmail.com
}

How to cite this paper: Oktem, H., Dilli, A., Kurkcuoglu, A. and Pelin, C. (2018) Prevalence of Septum Pellucidum Variations: A Retrospective Study. Open Access Library Journal, 5: e5017. https://doi.org/10.4236/oalib.1105017

Received: November 1, 2018

Accepted: November 23, 2018

Published: November 26, 2018

Copyright (c 2018 by authors and Open Access Library Inc.

This work is licensed under the Creative Commons Attribution International License (CC BY 4.0).

http://creativecommons.org/licenses/by/4.0/

\section{c) (7) Open Access}

\begin{abstract}
Background: The septum pellucidum is a vertical double membrane, extending from the lower surface of the corpus callosum to the fornix. Cavum septi pellucidi (CSP) is a cavity formation between the two membranes that separate the septum pellucidum from the anterior horn of the lateral ventricle. Cavum vergae $(\mathrm{CV})$ is the extension of the cavity between the two leaflets posterior to the splenium of the corpus callosum. In addition, another cystic variation, cavum velum interpositum (CVI), can be found at the level of crus fornices and corpus pineale. The aim of the present study was to retrospectively determine the type and prevalence of septum pellucidum variations in the adult population using Magnetic resonance imaging (MRI). Methods: Using a retrospective study design, the presence of septum pellucidum variations was investigated in 3128 patients aged $18-80$ years. The variations were defined as either CSP or CV on sagittal, axial, coronal T1- and T2-weighted images. In addition, the presence of $\mathrm{CV}$ and septum pellucidum agenesis was evaluated. Results: No variation was found in the septum pellucidum in 93.82\% of cases. CSP was the most frequent variation with a prevalence of $3.7 \%$. CV had a prevalence of 3.1\%. Apart from these variations, CVI was observed in $1 \%$ of all cases. Conclusion: Embryological development of the septum pellucidum and the neighboring anatomical structures occurs simultaneously. The authors believe that the present study might serve as a preliminary study for other clinical studies related to these variations.
\end{abstract}

\section{Subject Areas}

Anatomy \& Physiology

\section{Keywords}

Septum Pellucidum, Magnetic Resonance Imaging, Cavum Septi Pellucidi, Cavum Vergae, Cavum Velum Interpositum 


\section{Introduction}

The septum pellucidum is a vertical double membrane consisting of white matter, extending from the corpus callosum down to the fornix. The septum pellucidum is lined by ependymal cells on each surface, and it forms the medial border of the lateral ventricles together with the fornix. This structure, which is usually $1-3 \mathrm{~mm}$ thick, contains septal nuclei. The septum pellucidum is a component of the limbic system and has an important role in the connections between the hypothalamus and hippocampus, amygdala, habenula, and reticular formation of the brain stem [1] [2] [3].

The septum pellucidum must be evaluated together with the fornix and corpus callosum due to their anatomic contiguity and common embryological origins with respect to the prenatal diagnosis of brain malformations and clinical approaches [4].

In the process of early fetal development, the growth of the cerebral hemispheres leads to the spring-like rapid expansion of the commissural bodies on the fine roof of the diencephalon in the interhemispheric cleft. Commissural bodies develop faster than the fornix in the caudal direction, and the splenium corporis callosi finally envelops the fornix. As the rostrum of the corporis callosi develops, it closes off the residual sulcus medianus telencephalic groove to form a closed pocket-like cavum septi pellucidi (CSP), which consists of an interhemispheric fissure between the corpus callosum and the fornix. In this period, the lamina terminalis appears as a thin structure, and a thicker lamina reuniens appears more prominent. The lateral walls of CSP develop from lamina reuniens. Its thick walls become thinner with the rapid development of the corpus callosum, fornix, and cerebral hemispheres. At the end of this process, CSP extends and stretches to form the septum pellucidum [4]-[9].

CSP becomes visible from the second trimester of pregnancy and obliterates between the 36 and 40 weeks of gestation. CSP is a well-known ultrasonographic landmark in the fetal period that can be observed in premature infants and in all fetuses. The fusion is sometimes completed by $3-6$ months after birth. It remains as a narrow potential cavity in the postnatal period. However, if fusion does not occur, a cavity called CSP is formed between the two leaflets of the septum pellucidum that separates the anterior horns of the lateral ventricles, which is not connected to the ventricular system. The septum pellucidum is a triangular membrane, and CSP is bounded anteriorly by the genu, superiorly by the anterior portion of the body of the corpus callosum, and inferiorly by the rostrum of the corpus callosum and anterior commissure. The formation of CSP is directly affected by the development of the corpus callosum and midline telencephalic structures [1] [4] [5] [7] [8] [10] [11].

Cavum vergae (CV) is the posterior extension of CSP, and this cavity extends beyond the columns of the fornix and foramen monro posterior to the splenium of the corpus callosum [4] [9] [11].

Cavum velum interpositum (CVI) is a potential space between the two layers 
of tela choroidea, which is located above the third ventricle. The superior layer of tela choroidea attaches to the lower surface of the column of the fornix and hippocampal commissure, and the inferior layer attaches to the roof of the third ventricle parallel to the fibers of the choroid plexus. It can be considered as the physiological dilatation of the cisterna velum interpositum [9] [11] [12] [13].

\section{Material and Methods}

Brain MRI scans of 3128 (995 males, 2133 females) cases aged 18 - 80 years, who were admitted to Diskapi Yildirim Beyazit Education and Research Hospital between 2014 and 2015, were reviewed. The average age was $48.29 \pm 18.39$ years in males and $45.17 \pm 16.45$ years in females.

Examinations were performed using a 1.5 T MRI unit (Philips Achiva, Philips Medical Systems, Eindhoven, Netherlands) with spine coil, in supine position. The cranial MRI protocol included sagittal T1-weighted turbo spin-echo (TSE) images (TR/TE, 400/9 ms; slice thickness/interslice gap, 4/0.4 mm and NEX, 3), sagittal T2-weighted TSE images (TR/TE, 3000/120 ms; slice thickness/interslicegap, 4/0.4 mm and NEX, 3), and axial T2-weighted GE, FFE images (TR/TE, 600/14 $\mathrm{ms}$; slice thickness/interslice gap, $4 / 0.4 \mathrm{~mm}$, flip angle $25^{\circ}$ and NEX, 3).

Retrospectively, the presence of septum pellucidum CSP, CV, and CVI in the sagittal, axial, and coronal sections of the brain on MR images was recorded [3]. The patients with space-occupying intracranial lesions and hydrocephalus and the patients who underwent previous surgery in this area due to various reasons were excluded from the study.

SPSS 17 (Statistical Package for the Social Science, Ver 17.00, Chicago IL, USA) was used for the analysis of data. Frequency distributions were examined and interpreted. This study was approved by Baskent University Medical and Health Sciences Research Committee (Project number: KA 15/05) and supported by Baskent University Research fund.

\section{Results}

The present study found no variation related to the septum pellucidum on the retrospective assessment of MRI scans of $93.82 \%$ of cases. This study was performed in the adult population, and no cases of absence of the septum pellucidum were identified among the 3128 cases evaluated.

Figure 1 shows the most commonly observed variation was CSP in the present study. Of all cases, 3.7\% had CSP shown in Figure 1 and 3.1\% had CV shown in Figure 2. Accordingly, the difference between the prevalence of CSP and CV was not significant. Figure 3 shows the cases of coexistent CSP and CV account for $1.63 \%$ of all cases.

The patients were divided into four groups according to the age range as 18 25, $26-40,41-60$, and $61-80$ years. When distribution of the patients according to the age range is evaluated, CSP was found to be more prevalent in the age group of $41-60$ years and less prevalent in the age group of $18-25$ years. 


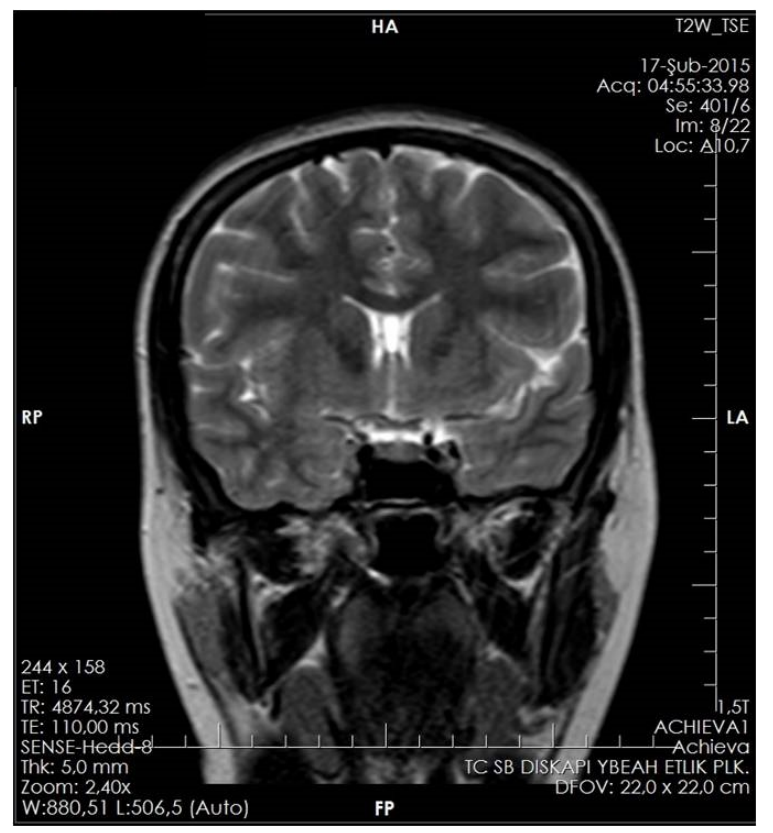

Figure 1. Coronal T2 weighted MRI of Cavum Septi Pellucidi (CSP).

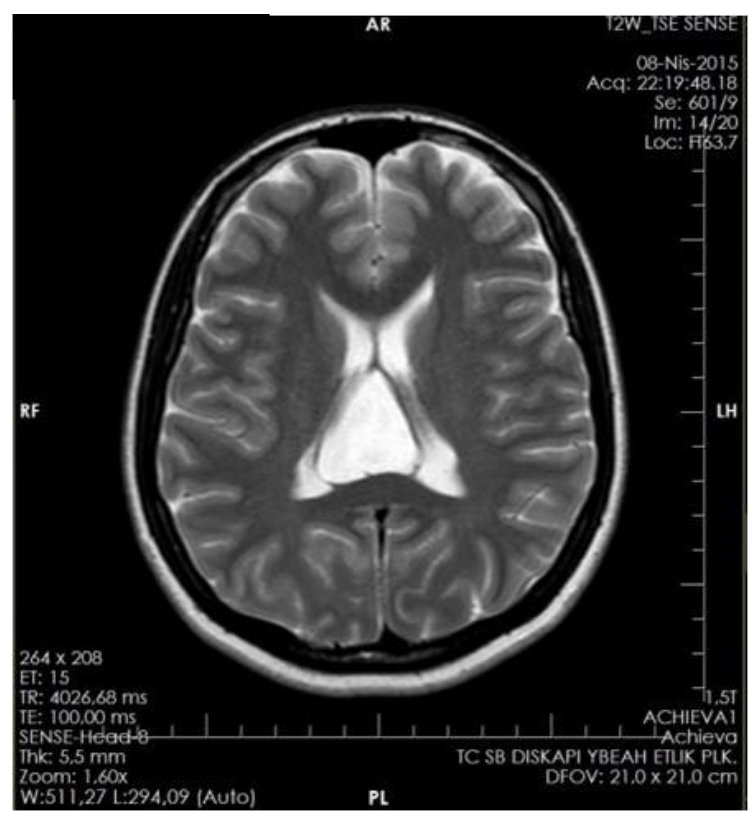

Figure 2. Axial T2 weighted MRI of Cavum Vergea (CV).

Of patients with coexistent CSP and CV, 49\% were in the age group of $41-60$ years (as demonstrated in Table 1).

In addition to these variations, CVI was observed in $1 \%$ of all cases. Table 2 demonstrates CVI was more prevalent in the age group of $61-80$ years.

\section{Discussion}

The development process of the septum pellucidum is synchronous with the development of limbic structures such as the corpus callosum, hippocampus, 


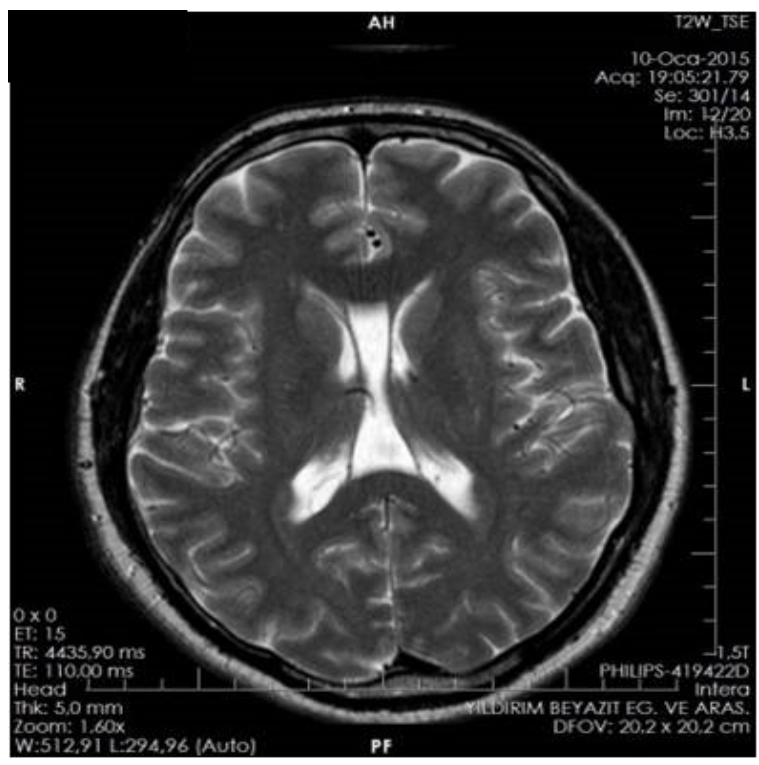

Figure 3. Axial T2 weighted MRI of coexistentCavum Septi Pellucidi (CSP) + Cavum Vergea (CV).

Table 1. The prevalence of CSP and CV in different age groups.

\begin{tabular}{cccccc}
\hline & \multicolumn{3}{c}{ CSP } & \multicolumn{3}{c}{ CV } \\
\hline Age (year) & $\mathbf{n}(\%)$ & Present & Absent & Present & Absent \\
\hline $\mathbf{1 8 - 2 5}$ & $436(13.9 \%)$ & $9(2.1 \%)$ & $427(97.9 \%)$ & $8(1.8 \%)$ & $428(98.2 \%)$ \\
$\mathbf{2 6 - 4 0}$ & $815(26.1 \%)$ & $23(2.8 \%)$ & $792(97.2 \%)$ & $20(2.5 \%)$ & $795(97.5 \%)$ \\
$\mathbf{4 1 - 6 0}$ & $1154(36.9 \%)$ & $55(4.8 \%)$ & $1099(95.2 \%)$ & $39(3.4 \%)$ & $1115(96.6 \%)$ \\
$\mathbf{6 1 - 8 0}$ & $723(23.1 \%)$ & $30(4.1 \%)$ & $693(95.9 \%)$ & $30(4.1 \%)$ & $693(95.9 \%)$ \\
Total & $3128(100 \%)$ & $117(3.7 \%)$ & $3011(96.3 \%)$ & $97(3.1 \%)$ & $3031(96.9 \%)$ \\
\hline
\end{tabular}

Table 2. The prevalence of CVI in different age groups.

\begin{tabular}{cccc}
\hline Age (year) & n (\%) & CVI & \\
\hline & & Present & Absent \\
\hline $\mathbf{1 8 - 2 5}$ & $436(13.9 \%)$ & $0(0.0 \%)$ & $431(98.9 \%)$ \\
$26-40$ & $815(26.1 \%)$ & $2(0.2 \%)$ & $800(98.2 \%)$ \\
$41-60$ & $1154(36.9 \%)$ & $11(1 \%)$ & $1107(95.9 \%)$ \\
$61-80$ & $723(23.1 \%)$ & $17(2.4 \%)$ & $688(95.2 \%)$ \\
Total & $3128(100 \%)$ & $30(\% 1 \%)$ & $3026(96.7 \%)$ \\
\hline
\end{tabular}

amygdala, and septal nuclei, which are located adjacent to the septum pellucidum. Therefore, it was suggested that anatomical variations detected in the septum pellucidum reflect embryonic developmental disorders in these afore-mentioned anatomical structures, such that, during fetal development, expansion of the hippocampus and the corpus callosum pushes the leaflets of the septum pellucidum and allows the leaflets to obliterate from posterior to anterior. It is assumed 
that dysgenesis of these neighboring structures leads to the persistence of CSP by disrupting the usual closure process of the septum pellucidum [4] [5] [8] [10]. Thus, many studies have been conducted on the variations and clinical significance of the septum pellucidum, but its clinical relevance remains unclear [4] [14].

Complete or partial agenesis of the septum pellucidum is observed in 2 - 3 individuals per 100,000 in the population, and it is typically associated with other intracranial abnormalities [6] [8] [15]. Agenesis of the septum pellucidum occurs in association with various congenital brain anomalies such as holoprosencephaly, septooptic dysplasia, schizencephaly, and agenesis of the corpus callosum [16]. In the cases reported by Supprian et al., two cases had isolated absence of the septum pellucidum without accompanying abnormalities, and the fornices were located more inferiorly than their usual position [15]. In the present study, no cases of the absence of the septum pellucidum were identified among the 3128 cases evaluated.

In a retrospective study by $\mathrm{Hsu}$ et al. using cranial ultrasonographic images of neonates, $93.6 \%$ had normal findings or normal variations that are considered insignificant findings, while CSP was the most common variation observed in $62.1 \%$ of the nenoates, and CV was less commonly observed with a prevalence of $1.19 \%$. They concluded that CSP obliterates in $85 \%$ of the cases at 3 - 6 months after birth and could therefore be considered a normal variation in newborn population [17].

The reported rates of CSP in healthy adults range from $0.1 \%$ to $85 \%$ [1] [3] [18] [19]. The reason for this wide range was linked to the developments in imaging methods, more frequent use of imaging methods, and use of thinner slices in these imaging methods that allows detection of small-sized pathologies. The prevalence of CSP in the present study was found to be 3.7\%. Many papers have considered that CSP could be related to the development of psychiatric disorders such as schizophrenia [1] [5] [20].

There is an insufficient number of studies on the prevalence of caval variations in healthy individuals; however, a prospective study conducted on a group of patients with neurological disorders reported that CV more commonly observed than CSP. There was no relationship between these anomalies and age of the patients [21]. The present study retrospectively evaluated MR images of the patients and did not consider clinical data in the comparison and therefore reported higher prevalence rates for CSP (3.7\%) than for CV (3.1\%).

Although CT scans revealed that the prevalence of CSP ranged from $0.15 \%$ to $28 \%$ in adult patients, the studies using MRI scans reported higher rates that ranged from $5.1 \%$ to $58.7 \%$. The prevalence of CSP in cadavers was found to be $22.96 \%$ in all cases, while the prevalence rate was $10.61 \%$ in the asymptomatic group [1]. In the present study, MRI scans revealed that the prevalence of CSP was found to be $3.7 \%$, which is lower than the reported prevalence rates.

In the study conducted on patients with schizophrenia, the prevalence of CSP was $23 \%$ in the patient group and $2 \%$ in the control group. It was concluded that 
CSP, CV, and partial agenesis of the corpus callosum were associated with brain anomalies responsible for the development of schizophrenia [20]. In addition, the prevalence of coexistent CSP and CV was $0.12 \%$.

In the study by Trzesniak et al. that evaluated the relationship between the first episode of psychosis and CSP, no significant difference was found between any CSP dimension and psychosis and control groups. However, they concluded that it was associated with the progression of the disease [10].

The prevalence of CSP was found to be $68.63 \%$ in the study by Filipovic B and Teofilovski Parapid, which was conducted on autopsy materials of patients with a neuropsychiatric disorder. The prevalence rate was $10.61 \%$ in the asymptomatic group. The overall prevalence rate was $22.96 \%$. In addition, CSP dimensions were significantly longer and wider in the symptomatic group [1]. In the present study, the prevalence of CSP on MRI scans was 3.7\%, a rate lower than that in the asymptomatic group. This suggests that cases remaining unrecognized by MRI scans could be detected during autopsy.

In the study by Silk et al., although there was no significant relationship between traumatic brain injury and CSP in pediatric patients compared to that in the control group, CSP was found to be wider in proportion to the degree of injury in the patient group [7]. In another study that evaluated traumatic brain injury and presence of CSP in adult patients, CSP was found to be significantly wider and longer in the patient group than that in the control group [19].

In the study by Akinola et al. that evaluated CT scans of 217 patients with a neurological disorder older than 6 months, the prevalence rates for CV, CVI, and CSP were $39.6 \%, 24.4 \%$, and $23 \%$, respectively. Although caval variations were more frequent among patients with a neurological brain disease, they were less common compared to patients with acquired lesions, which are caused by congenital brain diseases. In their study, CV was the most prevalent and CSP was the least prevalent caval variation. Besides, CSP was the less frequent cava variation [21]. However, in the present study, CSP was observed more frequently than CV.

In the study by Riedl et al. that evaluated 68 patients with optic nerve hypoplasia, 30 patients had septum pellucidum anomaly accompanying septo-optic dysplasia, 22 patients had absent septum pellucidum, and 8 patients had partial absence of septum pellucidum. Findings related to the septum pellucidum and hypoplasia of the corpus callosum on MRI scans are observed in $43 \%$ of the patients, and these findings are equally distributed among septum pellucidum groups. Cortical malformations are frequently observed in patients without the septum pellucidum [14].

Arachnoid cyst and meningioma are pathological anomalies originating from the velum interpositum. Arachnoid cysts of velum interpositum can be confused with CVI. The major characteristic of CVI is that it communicates with the quadrigeminal cistern. Another anomaly occurring in the velum interpositum and differentiating from the arachnoid cyst is that it originates from the quadrigeminal cistern [13]. 
The prevalence rates reported for septum pellucidum variations vary in a wide range in the literature. Embryological development of the septum pellucidum and the neighboring anatomical structures occurs simultaneously. Therefore, it can be argued that anatomical variations in the septum pellucidum might reflect possible embryonic developmental disorders in adjacent anatomical structures. The authors believe that the present study might serve as a preliminary study for their future studies and other clinical studies related to these variations.

\section{Acknowledgements}

The authors are thankful to Hulya Olmus for statistical analysis.

\section{Conflicts of Interest}

It is declared from all authors that there is no any conflict interest.

\section{References}

[1] Filipović, B. and Teofilovski-Parapid, G. (2004) Linear Parameters of Normal and Abnormal Cava Septi Pellucidi: A Post-Mortem Study. Clinical Anatomy, 17, 626-630. https://doi.org/10.1002/ca.20014

[2] Standring, S. (2005) Gray's Anatomy. 39th Edition, Philadelphia.

[3] Takahashi, T., Suzuki, M., Hagino, H., Niu, L., Zhou, S.Y., Nakamura, K., Tanino, R., Kawasaki, Y., Seto, H. and Kurachi, M. (2007) Prevalence of Large Cavum Septi Pellucidi and Its Relation to the Medial Temporal Lobe Structures in Schizophrenia Spectrum. Progress in Neuro-Psychopharmacology and Biological Psychiatry, 31, 1235-1241. https://doi.org/10.1016/j.pnpbp.2007.04.019

[4] Griffiths, P.D., Batty, R., Reeves, M.J. and Connolly, D.J. (2009) Imaging the Corpus Callosum, Septum Pellucidum and Fornix in Children: Normal Anatomy and Variations of Normality. Neuroradiology, 51, 337-345. https://doi.org/10.1007/s00234-009-0506-y

[5] Azab, W.A., Salaheddin, W., Alsheikh, T.M., Nasim, K. and Nasr, M.M. (2014) Colloid Cysts Posterior and Anterior to the Foramen of Monro: Anatomical Features and Implications for Endoscopic Excision. Surgical Neurology International, 5, 124. https://doi.org/10.4103/2152-7806.138364

[6] Falco, P., Gabrielli, S., Visentin, A., Perolo, A., Pilu, G. and Bovicelli, L. (2000) Transabdominal Sonography of the Cavum Septum Pellucidum in Normal Fetuses in the Second and Third Trimesters of Pregnancy. Ultrasound in Obstetrics \& Gynecology, 16, 549-553. https://doi.org/10.1046/j.1469-0705.2000.00244.x

[7] Silk, T., Beare, R., Crossley, L., Rogers, K., Emsell, L., Catroppa, C., Beauchamp, M. and Anderson, V. (2013) Cavum Septum Pellucidum in Pediatric Traumatic Brain Injury. Psychiatry Research: Neuroimaging, 213, 186-192. https://doi.org/10.1016/j.pscychresns.2013.03.001

[8] Sundarakumar, D.K., Farley, S.A., Smith, C.M., Maravilla, K.R., Dighe, M.K. and Nixon, J.N. (2015) Absent Cavum Septum Pellucidum: A Review with Emphasis on Associated Commissural Abnormalities. Pediatric Radiology, 45, 950-964. https://doi.org/10.1007/s00247-015-3318-8

[9] Yokota, A. (1993) Cysts Originating from a Defect in the Hemispheric Cleavage (Cavum Septi Pellucidi, Cavum Vergae, Cavum Veli Interpositi). In: Raimondi, A.J., 
Choux, M. and Di Rocco, C., Eds., Intracranial Cyst Lesions (Chapter 6) Part of the Series Principles of Pediatric Neurosurgery, Springer, New York, 87-99. https://doi.org/10.1007/978-1-4615-7281-7_6

[10] Trzesniak, C., Schaufelberger, M.S., Duran, F.L., Santos, L.C., Rosa, P.G., McGuire, P.K., Murray, R.M., Scazufca, M., Menezes, P.R., Hallak, J.E., Crippa, J.A. and Busatto, G.F. (2012) Longitudinal Follow-Up of Cavum septum Pellucidum and Adhesio Interthalamica Alterations in First-Episode Psychosis: A Population-Based MRI Study. Psychological Medicine, 42, 2523-2534. https://doi.org/10.1017/S0033291712000839

[11] Tubbs, R.S., Krishnamurthy, S., Verma, K., Shoja, M.M., Loukas, M., Mortazavi, M.M. and Cohen-Gadol, A.A. (2011) Cavum Velum Interpositum, Cavum Septum Pellucidum, and Cavum Vergae: A Review. Child s Nervous System, 27, 1927-1930. https://doi.org/10.1007/s00381-011-1457-2

[12] Chen, C.Y., Chen, F.H., Lee, C.C., Lee, K.W. and Hsiao, H.S. (1998) Sonographic Characteristics of the Cavum Velum Interpositum. American Journal of Neuroradiology, 19, 1631-1635.

[13] Funaki, T., Makino, Y., Arakawa, Y., Hojo, M., Kunieda, T., Takagi, Y., Takahashi, J.C. and Miyamoto, S. (2012) Arachnoid Cyst of the Velum Interpositum Originating from Tela Choroidea. Surgical Neurology International, 3, 120. https://doi.org/10.4103/2152-7806.102334

[14] Riedl, S., Vosahlo, J., Battelino, T., Stirn-Kranjc, B., Brugger, P.C., Prayer, D., Müllner-Eidenböck, A., Kapelari, K., Blümel, P., Waldhör, T., Krasny, J., Lebl, J. and Frisch, H. (2008) Refining Clinical Phenotypes in Septo-Optic Dysplasia Based on MRI Findings. European Journal of Pediatrics, 167, 1269-1276. https://doi.org/10.1007/s00431-007-0666-x

[15] Supprian, T., Sian, J., Heils, A., Hofmann, E., Warmuth-Metz, M. and Solymosi, L. (1999) Isolated Absence of the Septum Pellucidum. Neuroradiology, 41, 563-566. https://doi.org/10.1007/s002340050805

[16] García-Arreza, A., García-Díaz, L., Fajardo, M., Carreto, P. and Antiñolo, G. (2013) Isolated Absence of Septum Pellucidum: Prenatal Diagnosis and Outcome. Fetal Diagnosis and Therapy, 33, 130-132. https://doi.org/10.1159/000338009

[17] Hsu, C.L., Lee, K.L., Jeng, M.J., Chang, K.P., Yang, C.F., Tsao, P.C., Lee, Y.S., Chen, S.J., Soong, W.J. and Tang, R.B. (2012) Cranial Ultrasonographic Findings in Healthy Full-Term Neonates: A Retrospective Review. Journal of the Chinese Medical Association, 75, 389-395. https://doi.org/10.1016/j.jcma.2012.06.007

[18] Degreef, G., Bogerts, B., Falkai, P., Greve, B., Lantos, G., Ashtari, M. and Lieberman, J. (1992) Increased Prevalence of the Cavum Septum Pellucidum in Magnetic Resonance Scans and Post-Mortem Brains of Schizophrenic Patients. Psychiatry Research, 45, 1-13. https://doi.org/10.1016/0925-4927(92)90009-S

[19] Gardner, R.C., Hess, C.P., Brus-Ramer, M., Possin, K.L., Cohn-Sheehy, B.I., Kramer, J.H., Berger, M.S., Yaffe, K., Miller, B. and Rabinovici, G.D. (2016) Cavum Septum Pellucidum in Retired American Pro-Football Players. Journal of Neurotrauma, 33, 157-161. https://doi.org/10.1089/neu.2014.3805

[20] Degreef, G., Lantos, G., Bogerts, B., Ashtari, M. and Lieberman, J. (1992) Abnormalities of the Septum Pellucidum on MR Scans in First-Episode Schizophrenic Patients. American Journal of Neuroradiology, 13, 835-840.

[21] Akinola, R.A., Idowu, O.E. and Nelson-Paseda, A.O. (2014) Caval Variations in Neurologically Diseased Patients. Acta Radiologica Short Reports, 3, 1-6. https://doi.org/10.1177/2047981614530288 